\title{
La circulation de l'eau dans la plante en flux non conservatif : quelques faits et problèmes
}

\author{
Non-conservative flux circulation of water \\ in the plant : facts and problems
}

\author{
Pierre Cruiziat \\ Station de Bioclimatologie CNRA, route de Saint-Cyr, 78000 Versailles
}

\section{Introduction}

Cet exposé complète celui d'A. Berger. On y trouvera succinctement présentés quelques faits et problèmes actuels concemant le fonctionnement hydrique du végétal du point de vue de la physiologie de la plante individuelle. On a choisi de mettre l'accent plutôt sur des questions non résolues et des problèmes de méthode tout en les situant, autant que faire se peut, dans un ensemble de faits plus généraux. Le lecteur ayant eu connaissance des autres communications de ces joumées consacrées à l'hydrodynamique humaine, se rendra aisément compte du décalage important qui existe entre l'état de nos connaissances dans ce domaine et celui de l'hydrodynamique végétale.

\section{Flux conservatif et flux non conservatif}

Avant d'expliciter ces termes dans le cas de la circulation de l'eau dans la plante, il est bon de rappeler quelques caractéristiques générales (Fig. 1 et photos d'anatomie)

Comme on l'a vu précédemment (Berger), il est généralement admis que l'eau circule du sol à travers la plante jusqu'à l'atmosphère le long de gradients de potentiel hydrique $\Delta \psi$. Le moteur essentiel de cette circulation est la transpiration, c'est-à-dire l'évaporation de l'eau, qui a lieu au niveau des feuilles. Les valeurs de celle-ci sont fonction d'une part de l'énergie arrivant sur les feuilles, et de la couche limite aérodynamique entourant la feuille, d'autre part des caractéristiques foliaires et en particulier de la résistance stomatique traduisant la plus ou moins grande ouverture des stomates ${ }^{(1)}$.

Du point de vue de la distance parcourue dans le végétal, le trajet de l'eau en phase liquide représente en général plus de $99 \%$ du trajet total de l'eau à travers la plante. Par contre du point de vue des chutes de potentiel, c'est le long du trajet en phase gazeuse, donc sur moins d'un mm habituellement, que la chute de potentiel est la plus importante. En effet, en prenant pour potentiel de l'eau des feuilles -- 10 bars $^{(2)}$ à $25^{\circ} \mathrm{C}$ (valeur très fréquente), l'humidité en équilibre est de $99,5 \%$ environ. Or dans les conditions naturelles, il est assez courant d'avoir $50 \%$ d'humidité relative en été par une belle journée, ce qui correspond à un $\Psi$ de - 952 bars à $25^{\circ} \mathrm{C}$. Ainsi dans les feuilles, entre les parois des cellules et l'air extérieur, on a un gradient de plusieurs centaines de bars sur un mm, alors que celui-ci est de l'ordre de une à deux dizaines entre le sol et les feuilles.

Un autre caractère distingue aussi très nettement les trajets en phase liquide et gazeuse : ce sont les sections de passage. Bien qu'il y ait très peu de données sur ce sujet, on peut estimer que les rapports Surface d'échange en phase vapeur sur Surface de conduction en phase liquide sont de lordre de 100 à 1000 si on prend la surface foliaire comme référence. Cependant l'essentiel de la perte en eau des feuilles se fait à travers les stomates qui ne représentent que 1 à $2 \%$ de la surface totale d'une feuille. De plus l'évaporation s'effectue en fait au niveau des surfaces internes des cellules foliaires, à l'intérieur de ce corps poreux que constitue une feuille. Or le développement de ces surfaces internes représente souvent plusieurs dizaines de fois la surface foliaire. De même pour la phase liquide, deux raisons font de la section transversale totale des vaisseaux du xylème une mauvaise mesure de la capacité réelle de conduction.

(1) Les stomates sont de minuscules $(5-30 \mu$ environ) trous aux faces supérieure et surtout inférieure des feuilles permettant les échanges gazeux (entrée du $\mathrm{CO}_{2}$, départ de la vapeur d'eau) des feuilles avec l'extérieur. Leur densité est de 10 à 300 au $\mathrm{mm}^{2}$ environ.

(2) 1 bar est équivalent à $101,3 \mathrm{~J} . \mathrm{Kg}^{-1}$. 

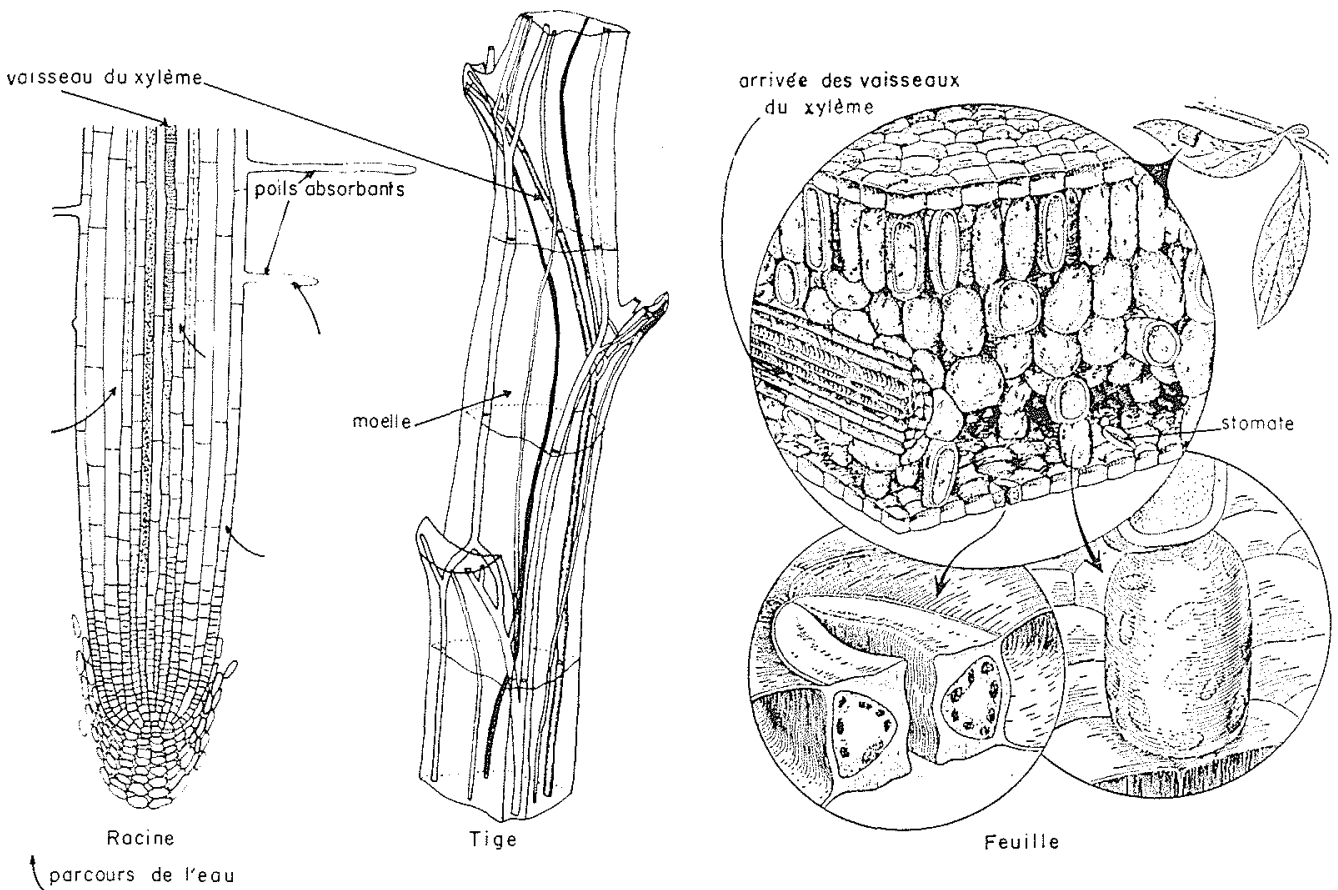

Figure 1 - Anatomie de la circulation de l'eau à travers la plante.

Ces trois photos montrent quelques aspects anatomiques de l'appareil conducteur du tournesol.

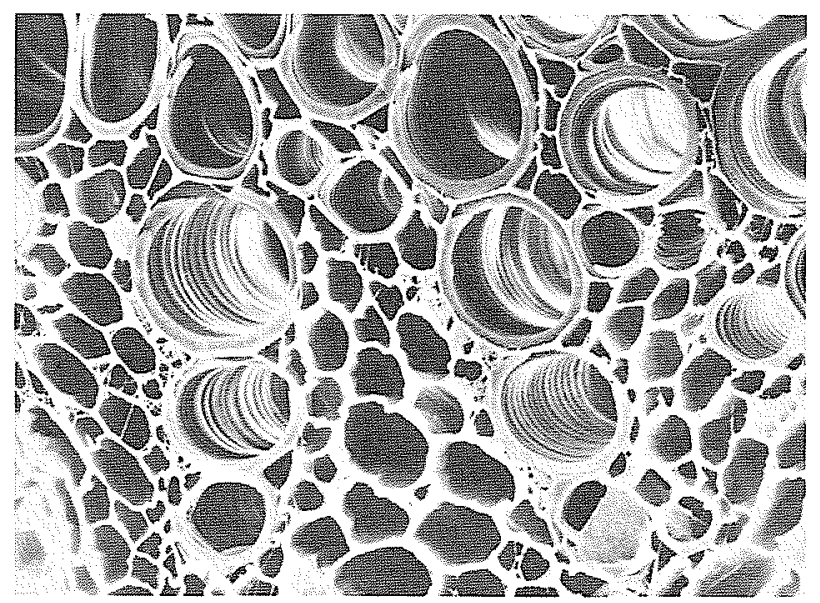

Photo A : coupe transversale dans un faisseau libéro-ligneux montrant plusieurs vaisseaux du xylène.

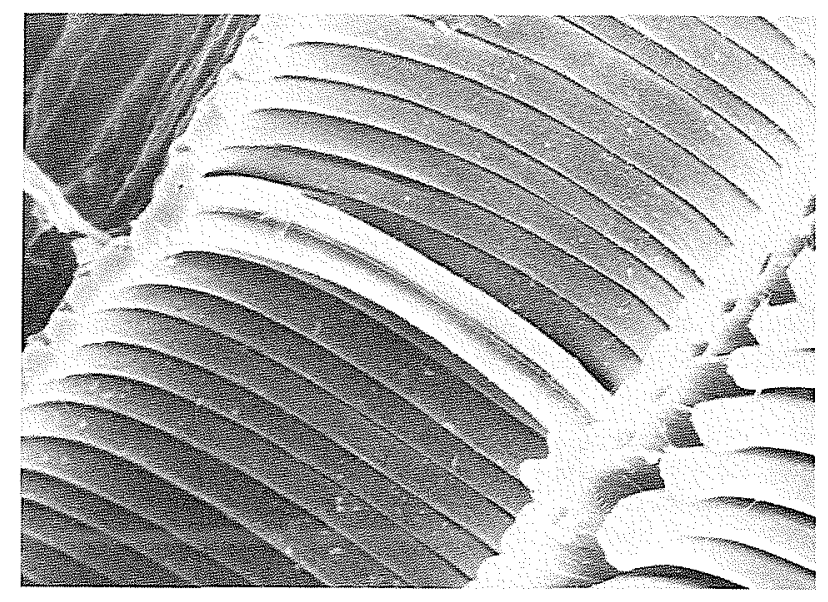

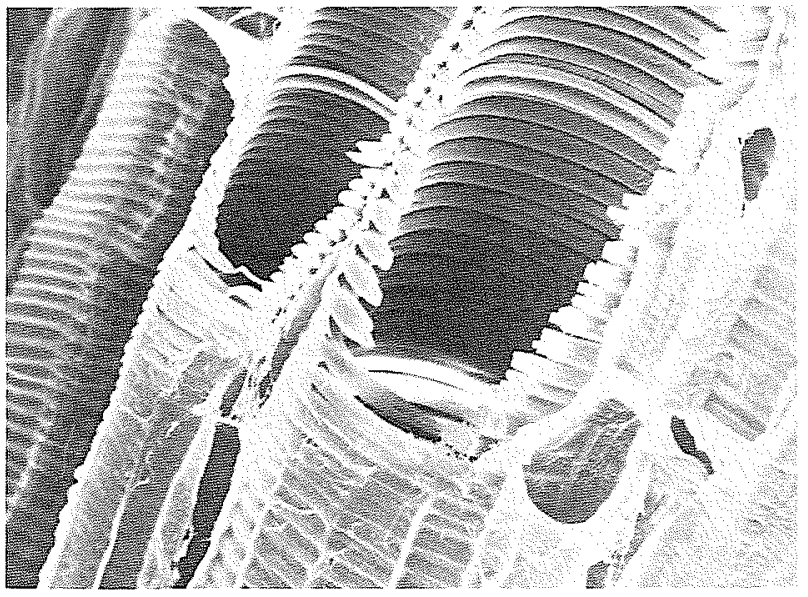

Photo B : coupe longitudinale dans un faisseau libero-ligneux montrant un groupe de vaisseaux.

Photo $\mathrm{C}$ : détail d'un vaisseau du xylème montrant l'ossature formée par les épaississements spiralés le lignine. 
La première, c'est que nous ne connaissons pas quel est le pourcentage de cette section qui est réellement conducteur spécialement chez les vieux arbres. La seconde, très importante, c'est qu'en raison de la loi de Poiseuille, le volume de liquide transitant dans des capillaires est proportionnel à la puissance quatre $\mathrm{du}$ rayon. Une même surface totale de capillaires peut donc véhiculer des quantités d'eau extrêmement différentes suivant le diamètre individuel de chaque capillaire. Il faut donc rester très prudent dans l'utilisation de ce genre de résultats visant à définir le rapport des sections de passage entre phase liquide et phase vapeur et bien savoir comment ils ont été établis.

Revenons maintenant à la distinction entre flux conservatif et flux non-conservatif. On peut en effet classer l'ensemble des conditions d'alimentation en eau d'un végétal pris globalement, en deux classes :

- Les conditions de flux conservatif qui correspondent aux moments où, sur l'intervalle de temps considéré, l'absorption et la transpiration sont égales ${ }^{(3)}$. L'état hydrique du végétal reste alors globalement fixe et la plante peut être assimilée à un circuit de résistances en série. On peut considérer ces conditions réalisées, soit sur des cycles de $24 \mathrm{~h}$, soit sur des intervalles plus courts de l'ordre de la dizaine de minutes à l'heure par exemple, suivant les conditions d'ETP en particulier ${ }^{(4)}$.

- Les conditions de flux non conservatif où sur l'intervalle de temps considéré, l'absorption et la transpiration ne sont pas égales. En période de forte évaporation, on assiste souvent le jour à un déssèchement plus ou moins important, la transpiration excédant l'absorption, et à une réhumectation la nuit où la situation inverse se produit. Par rapport au cas précédent, un nouvel élément caractérisant la plante du point de vue hydrique apparait : les variations de teneur en eau des différents organes, qui peuvent s'analyser comme la mise en œuvre de réservoirs, qui ne sont pas autre chose au niveau élémentaire que les cellules des différents tissus de la plante.

Pour expliquer l'établissement d'un déficit hydrique chez un végétal, donc le passage aux conditions de flux non conservatif, on trouve encore assez souvent écrit des phases du genre ci-dessous dont l'origine remonte probablement à l'article bien connu de Kramer (1938) : "Pendant le jour, l'absorption d'eau à travers les racines acquiert un certain retard par rapport à la transpiration du fait des résistances au transfert de l'eau à travers la plante". Il s'agit là d'une explication pour le moins incomplète sinon incorrecte. Elle reflète cependant bien l'attitude qui prévaut aujourd'hui et qui consiste à considérer ce phénomène comme tout à fait naturel (ce qui est exact) et clair (ce qui l'est moins). On peut alors foumir la seconde explication suivante,cf. Fig. 2,(Jarvis 1975) qui pourrait être satisfaisante si la plante n'était réellement qu'un système de réservoirs - au sens des

(3) Pour être totalement rigoureux, il faudrait ajouter "en conditions de croissance nulle". Car autrement, l'augmentation de la matière sèche due à celle-là suppose, pour que la teneur en eau reste la même dans tout le végétal, une absorption un peu supérieure à la transpiration.

(4) ETP $=$ évapotranspiration potentielle.

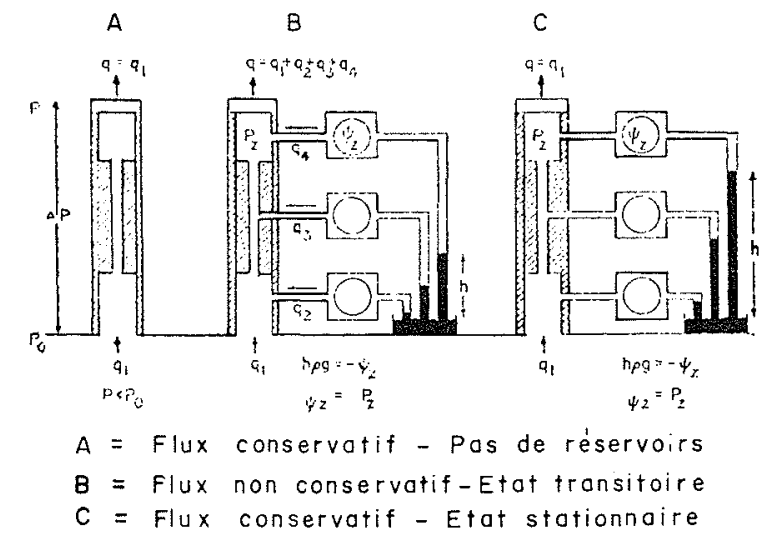

D

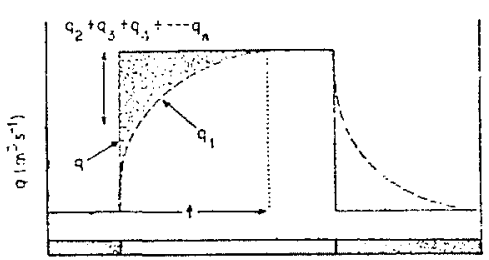

Figure 2 - Explication schématique de l'établissement d'un certain déficit dans la plante. A) Il n'y a pas de réservoir : la plante est analogue à un tuyau dont la section varie suivant l'organe considéré. B) La plante a des réservoirs : lorsqu'elle transpire, les cellules proches des vaisseaux du xylème est initialement en équilibre avec eux, se trouvent alors à un potentiel plus haut ; elles débitent donc jusqu'à l'établissement d'un nouvel equilibre. C) entre elles et le xylème, $q$ est la perte d'eau par transpiration, $q_{1}$ l'absorption d'eau par les racines et $q_{2} \ldots q_{4}$ les flux d'eau sortant des "réservoirs" constitués par les cellules. $P_{\mathrm{z}}$ est le potentiel dans le xylème et $\Psi_{\mathrm{z}}$ celui des cellules adjacentes à la hauteur $z$. D) Flux d'eau à travers la plante lorsque la transpiration est brusquement augmentée par rayonnement par exemple. La ligne continue représente la perte par transpiration $q ; q_{1}$ l'absorption des racines et $q_{2} \ldots q_{n}$ les différents flux de sortie des réservoirs (d'après Jalvis, 1975).

hydrauliciens - alimenté d'un côté et débitant par l'autre : les changements de teneur en eau dans une plante sont provoqués par le mouvement de l'eau depuis les régions de haut potentiel vers les régions à potentiel plus bas du xyleme. Ainsi le potentiel des tissus suit celui des vaisseaux du xylème habituellement avec un certain retard dû aux caractéristiques de stockage des tissus. Le "retard", c'est-à-dire l'inégalité à un moment donné, entre l'absorption et la transpiration, ne peut être dû à la seule présence de résistances (le long d'un tuyau, même comprenant des rétrécissements, le volume d'entrée est égale à celui qui sort), mais ne peut se produire au contraire que parce qu'il y a capacité de stockage. Malheureusement un végétal présente par rapport à l'analogie hydraulique précédente de sérieuses différences dont les deux principales paraissent être les suivantes.

a) L'écoulement de l'eau dans ce système se fait à contregravité, le "moteur" - l'évaporation - étant placé au point haut ${ }^{(5)}$.

B) La continuité hydraulique à travers tout le système est assurée par un réseau complexe de capillaires

(5) Mise à part certaines périodes où la poussée radiculaire joue un rôle prépondérant dans la montée de la sève chez les plantes herbacées. 
et de cellules dont les parois mouillables ne sont ni rigides ni imperméables.

La théorie de la tension-cohésion (Dixon, 1914) fournit un cadre général d'explication de la circulation de l'eau dans la plante tenant compte de ces deux points précédents en particulier. Mais elle n'explicite rien dans le détail et reste purement qualitative ; mis à part les problèmes de cavitation qui seront effleurés un peu plus loin, bien des questions restent obscures, du type de celle-ci : peut-on expliquer quantitativement les rapports existants entre l'évaporation et la tension dans les vaisseaux du bois qui sont séparés des surfaces évaporantes par plusieurs cellules ? Comment se fait-il que ces cellules intermédiaires ne soient pas déshydratées plus que d'autres et comment transmettent-elles cette tension ? Comment s'initie cette tension ? Là encore,le fait de dire : "une feuille dont les stomates sont bien ouverts évapore comme une surface d'eau libre (toutes choses étant égales par ailleurs)" n'est vrai que sous certains aspects. Car lorsqu'une telle surface évapore, il ne s'en suit pas la formation de tension au sein de la masse liquide sous-jacente. Il faut donc tenir compte aussi du fait que l'évaporation dans la feuille a lieu au niveau d'un corps poreux mouillable et que lorsque les limites du liquide régressent en deça de celles du support, les surfaces mouillées sont susceptibles d'exercer une force sur le liquide conduisant à une tension opposée dans celui-ci. A supposer que cette description soit correcte, elle n'en laisse pas moins subsister bien des interrogations. Il est curieux de constater que dans le nombre important d'articles et d'ouvrages consacrés aux relations eau-plante, ces aspects - peut-être bien mieux connus dans d'autres disciplines - ne sont pour ainsi dire jamais abordés !

\section{Les réserves en eau du végétal}

Les tissus d'une plante peuvent être regardés comme de nombreux réservoirs reliés les uns aux autres en parallèle et en série par rapport au sol. Ainsi le flux total de transpiration d'une plante est-il la somme d'un grand nombre de flux partiels provenant des diverses parties de la plante et de celui provenant du sol :

$$
T=A+\sum_{i=1}^{i=n} q_{i}
$$

avec $q_{i}=\left(\psi_{i}-\psi_{x}\right) / R_{i}$ où $\psi_{i}=f\left(h_{i}\right)$

$T=$ transpiration ou perte en eau de la plante

$A=$ absorptions des racines (flux d'entrée de l'eau du sol dans la plante)

$q_{i}=$ différents flux provenant des diverses parties de la plante

$\psi_{x}=$ potentiel dans le circuit principal de la transpiration

$R_{i}=$ résistance entre le réservoir considéré et le circuit principal

$\Psi_{i}=$ potentiel hydrique du réservoir $i$

$h_{i}=$ teneur en eau relative $=$ poids frais-poids sec/poids frais à la pleine turgescence - poids sec.
Le volume d'eau qui peut sortir d'un tissu est donc entre deux instants

$$
\Delta V_{i}=V_{i} \Delta h_{i}=\int_{t_{1}}^{t_{2}} q_{i} d t
$$

$V_{i}$ étant le volume d eau correspondant à la teneur relative en eau $h_{i}$.

Ces quantités sont difficiles à apprécier faute de méthodes adéquates.

L'importance relative des flux provenant des réservoirs à chaque instant et leur mode de fonctionnement dépend d'au moins trois facteurs : leur capacité maximale, la relation liant leur potentiel et leur état hydrique, la résistance les séparant des vaisseaux du bois. Là encore, et même en supposant que chaque organe puisse être considéré comme une unité fonctionnelle homogène - ce qui nest pas le cas au moins pour les feuilles comme nous le verrons plus loin - nos connaissances sont très faibles, faute de techniques appropriées. Pour les feuilles, la relation potentiel-teneur en eau est assez bien connue. Elle varie beaucoup d'une espece à l'autre et même en particulier suivant l'âge de la feuille chez une même plante. C'est l'une des raisons pour lesquelles le tournesol, le tabac, le chou, en période de dessèchement, présentent habituellement des feuilles inférieures (les plus vieilles) plus flétries que les supérieures (les plus jeunes) : une même chute de potentiel entraine une faible variation de teneur en eau chez les feuilles jeunes, mais une forte variation de teneur en eau chez les plus âgées.

On pourrait s'attendre à ce qu'en régime de dessè. chement, ce soient les réservoirs des tissus les plus voisins des surfaces de transpiration qui perdent leur eau les premiers et qu'ensuite, progressivement, le complément de l'absorption soit foumi par les autres organes plus éloignés. En fait, comme on vient de le dire, cela dépend des caractéristiques des différents végétaux. Chez des plantes comme le coton, le tournesol, le haricot, ce n'est pas, semble-t-il, de haut en bas que la vague de deshydratation se propage : sous l'effet de la transpiration et par l'intermédiaire du système vasculaire, l'eau répartie dans les différentes parties de la plante est sollicitée quasiment instantanément et c'est, perpendiculairement à ces axes privilégiés de conduction que constituent les vaisseaux, que la deshydratation va se faire. La "disponibilité en eau d'un organe" ne dépend donc pas obligatoirement de sa place par rapport à la sortie de l'eau, ni même de la teneur en eau de l'organe (Huck et Coll., 1970 ; Cruiziat, 1972). Par contre, chez les arbres, un certain nombre d'auteurs ont constaté qu'au contraire il y avait, à partir du lever du soleil, une "vague" de deshydratation descendant lentement le long du tronc et le soir une "vague" de réhydratation commençant par le bas.

Donnons maintenant quelques éléments chiffrés sur l'ordre de grandeur des "réserves en eau" que constituent les différents organes d'une plante. Ces éléments sont de deux sortes : dans les premiers (tableau I) tirés de Jarvis 1975, les réserves sont exprimées en heures de transpiration moyenne ce qui signifie, par exemple (1ère ligne), que chez une plante herbacée en culture 


\section{Tableau I}

Estimation des réserves en eau des différents organes de plantes herbacées en culture ou de jeunes conifères en plantation dense (d'après Jarvis, 1975), exprimées en heures de transpiration.

\begin{tabular}{|l|c|c|}
\hline & Culture herbacée & $\begin{array}{c}\text { Peuplement } \\
\text { de conifères }\end{array}$ \\
\hline $\begin{array}{l}\text { Feuilles } \\
\text { Racines }\end{array}$ & 0,3 & 1 \\
$\begin{array}{l}\text { Tige ou } \\
\text { Tronc : } \\
\text { tissus } \\
\text { vivants } \\
\text { tissus non } \\
\text { vivants }\end{array}$ & 1,3 & 14 \\
\hline
\end{tabular}

flux qu'elle perd par transpiration. Elle ne peut donc "tenir seule" longtemps --sous peine de se deshydrater irréversiblement et de mourir - si elle ne peut tirer du sol des quantités voisines de celles qu'elle transpire.

- Quand une plante se flétrit (ou se réhumecte) tous les organes sont touchés mais de façon très inégale.

- Les racines peuvent constituer des réserves en eau supérieures à celles des feuilles. De plus ce dessèchement des racines à deux conséquences : d'une part il s'accompagne d'une réduction importante (30 à $50 \%$ de leur diamètre ce qui augmente donc la distance sol-racines et par là, la résistance au passage de l'eau du sol vers les racines ; d'autre part si, a chaque instant l'eau perdue par transpiration provient tout autant des feuilles que des racines, cela signifie que les gradients de potentiel s'établissent non pas seulement en fonction de la distance par

\begin{tabular}{|c|c|c|c|}
\hline \multicolumn{4}{|r|}{ Tableau II (d' } \\
\hline \multicolumn{4}{|c|}{ déficit moyen $d^{P}=21,6 \%$} \\
\hline & $\frac{w_{T}}{w_{T}^{P}}$ & $d$ & $x$ \\
\hline$F$ & \begin{tabular}{c}
\multicolumn{2}{c}{0,36} \\
$0,31 \quad 0,40$
\end{tabular} & $\begin{array}{c}15,8 \% \\
10,2 \quad 20,2\end{array}$ & $\begin{array}{c}0,27 \\
0,14 \quad 0,38\end{array}$ \\
\hline$T$ & $0,12^{0,16} 0,26$ & $\begin{array}{c}10,0 \% \\
5,3 \quad 15,3\end{array}$ & \begin{tabular}{cc}
\multicolumn{2}{c}{0,07} \\
0,06 & 0,09
\end{tabular} \\
\hline$R$ & \begin{tabular}{cc}
\multicolumn{2}{c}{0,48} \\
$0,43 \quad 0,53$
\end{tabular} & $\begin{array}{c}30,6 \% \\
22,8 \quad 43,5\end{array}$ & \begin{tabular}{c}
\multicolumn{2}{c}{0,66} \\
$0,56 \quad 0,80$
\end{tabular} \\
\hline
\end{tabular}

Haricot

\begin{tabular}{|c|c|c|c|}
\hline \multicolumn{4}{|c|}{ déficit moyen $d^{P}=23,8 \%$} \\
\hline & $\frac{W_{T}}{W_{T}^{P}}$ & $d$ & $x$ \\
\hline$F$ & 0,38 & $21,8 \%$ & 0,36 \\
\hline & $0,37 \quad 0,39$ & $17,0 \quad 31,0$ & $0,31 \quad 0,39$ \\
\hline$T$ & 0,05 & $9,6 \%$ & 0,02 \\
\hline & $0,04 \quad 0,05$ & $5,0 \quad 19,0$ & $0,01 \quad 0,04$ \\
\hline$R$ & 0,57 & $25,5 \%$ & 0,62 \\
\hline & 0,58 & 26,0 & $0,60 \quad 0,67$ \\
\hline
\end{tabular}

Poids relatif en eau $\left(W_{T} / W_{T}^{P}\right)$, déficit $(d)$ et contribution $(X)$ de l'organe considéré (feuilles, tige, racines) à la perte totale en eau d'un pied ayant conduit à un certain déficit global $d^{P} ;$ voir le texte pour plus d'explications.

n'absorbant aucun flux d'eau en provenance du sol, les feuilles pourraient subvenir à environ 0,3 heure de transpiration moyenne, ou à 1 heure pour le cas d'un jeune conifère. Il s'agit là, il faut le souligner, uniquement d'estimations calculées et non de mesures. Dans les seconds (Tableau II) au contraire sont donnés des résultats de mesure obtenus en laboratoire, les réserves en eau de chaque organe (désignées par $X$ ) sont exprimées en \% de l'eau perdue par toute la plante durant un dessèchement. Ainsi par exemple on lit dans ce tableau que pour des tournesols ayant subi un dessèchement conduisant à un déficit moyen(6) au niveau de toute la plante de $23,8 \%$, les feuilles ont fourni $36 \%$ de la quantité totale d'eau perdue par la plante, la tige $2 \%$ et les racines $62 \%$.

Il faut retenir de ces donnés trois points :

- En général (sauf pour les arbres adultes) "l'autonomie hydrique" d'une plante annuelle est faible devant le

(6) Le déficit en eau d'un organe ou d'une plante correspond au rapport de la teneur en eau du déficit donné sur la teneur maximale en eau appelée pleine turgescence. rapport aux feuilles mais plus encore en fonction de la distance par rapport aux voies principales de la conduction liquide que constituent les vaisseaux du bois.

\section{Deux exemples d'approche du fonctionnement hydrique des feuilles.}

\section{Les cinétiques de réhumectation.}

Il est intéressant de connaître la durée de réhumectation d'une feuille et comment s'effectue cette réhumectation. On peut pour cela procéder de la façon suivante : on fait subir à une feuille de Tournesol isolée, munie de son pétiole et préalablement portée à la pleine turgescence, un dessèchement lui conférant un déficit de saturation donné. On la place alors dans une enceinte obscure dont l'atmosphère est saturée en vapeur d'eau, la base du pétiole bouchée à la cire, plongeant dans l'eau d'un cristallisoir. La feuille est par ailleurs suspendue au crochet d'une balance enregistreuse (cf. Fig. 3). Le débit 


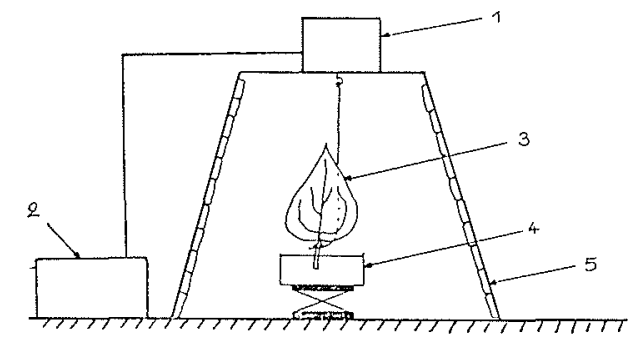

Figure 3 - Dispositif expérimental pour les cinétiques de réhumectation. 1 - balance enregistreuse ; 2 - traceur de courbe ; 3 -- feuille sur son support ; 4 - cristallisoir rempli d'eau pure; 5 - chambre de réhumectation tapissée d’éponges humides.

de la réhumectation commence au moment où, avec une lame de rasoir neuve, on coupe rapidement l'extrêmité inférieure du pétiole sous l'eau. Du fait de l'existence d'une différence de potentiel entre l'eau de la feuille et celle du cristallisoir, l'eau monte dans la feuille jusqu'à ce que l'égalité des potentiels soit réalisée. L'augmentation de poids de la feuille lue à chaque instant sur la balance permet de connaître la quantité d'eau stockée dans la feuille à chaque instant de la réhumectation. (La transpiration est tout à fait négligeable étant donnée les conditions de saturation de l'enceinte). On peut donc obtenir, en fonction du temps, la variation du flux pénétrant dans la feuille à chaque instant, c'est-à-dire sa cinétique de réhumectation. L'étude de celle-ci permet de tirer une certain nombre de renseignements concernant le fonctionnement hydrique de la feuille en régime de réhumectation.

La phase 1 (Fig. 4A à C) correspondrait à l'entrée de l'eau dans les vaisseaux du xylème et peut-être aussi dans les cellules adjacentes associées constituant une partie des faisceaux libéroligneux. Le fait que la pente de cette phase ne soit pas infinie montre que ces structures ont elles-mêmes une certaine contenance et qu'elles se réhumectent. Le volume correspondant est d'environ 16$19 \%$ du volume total de l'eau absorbée au cours de la réhumectation. Cependant, comment se fait-il qu'elle ne soit pas toujours présente? On peut invoquer plusieurs explications : soit une durée trop brève rendant cette phase indétectable par la technique employée, soit une pente se confondant avec celle de la phase suivante, soit enfin l'état de cavitation des vaisseaux. On sait en effet depuis longtemps que dans les vaisseaux du xyleme, l'eau est habituellement sous tension du fait de la transpiration. Cette tension correspond à un état instable. Bien qu'à 1'heure actuelle nos connaissances sur cet état soient très faibles, il semble probable que, lors du dessèchement de feuilles isolées, des phénomènes de cavitation se produisent dans les vaisseaux (Milbum, 1966, 1973 ; West et Gaff, 1976) qu'il s'agisse de rupture de colonnes liquides ou de formation de bulles à partir de germes contenus dans la sève.Milburn a procédé comme suit. Il suspend (par son pétiole) une feuille de Ricin (Ricinus sp.) par l'intermédiaire d'une fine aiguille d'acier à un support. Un petit détecteur soudé à cette aiguille lui permet d'enregistrer, à l'aide d'un montage adéquat, les ultrasons produits lors de la cavitation. La feuille est alors illuminée de façon à transpirer. Il se produit alors un certains nombre d'ultrasons, se traduisant grâce au montage, en bruits audibles saccadés (des "clicks") dus selon Milburn au fait que l'eau des vaisseaux du xylème cavite. Il enregistre alors ces "clicks" au cours du dessèchement de la feuille et trouve des courbes du genre de celle de la figure 5. Les autres résultats importants qu'il a obtenus sont les suivants :

- Si le même traitement précédent (dessèchement sous une lampe) est appliqué à une feuille d'Elodea canadensis qui ne possède pas de vaisseaux du bois, on n'enregistre pas de "clicks".

- Il y a une relation assez nette entre les valeurs des intensités de transpiration et l'importance de la cavitation.

- Si pendant la réhumectation d'une feuille on ajoute brusquement à la solution dans laquelle son pétiole plonge de fines particules de carbone, on remarque un grand pic de "clicks" puis la plante se flétrit.

- Si l'on soumet la vascularisation d'une feuille, préalablement desséchée et dont le pétiole plonge dans l'eau d'un cristallisoir, à un certain vide (par l'intermédiaire d'une pompe à vide), de nombreuses bulles sortent par l'extrêmité du pétiole ; la vitesse de réhumectation après ce traitement est supérieure à ce qu'elle était, ce qui montre bien qu'on a ainsi augmenté la conduction dans les vaisseaux.
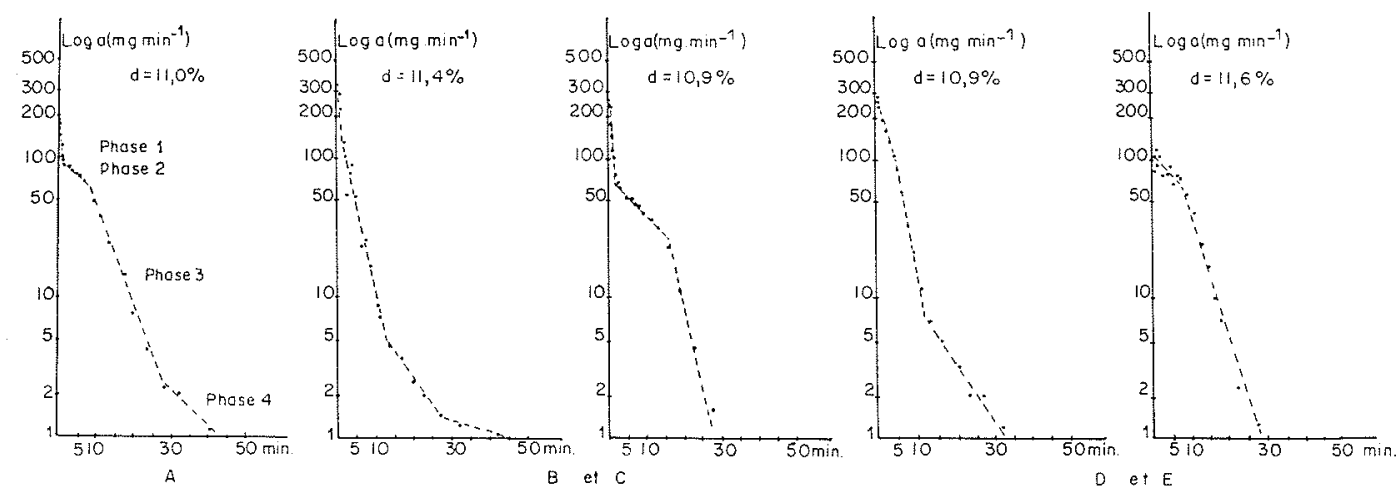

Figure 4 - Exemples de courbes expérimentales obtenues à partir de feuilles à $10 \%$ de déficit initial (d). A) Courbe typique de la classe I montrant les différentes phases. B et C) Deux cas "extrèmes" de la classe I. D et E) Deux exemples de la classe II : la phase I est absente. 


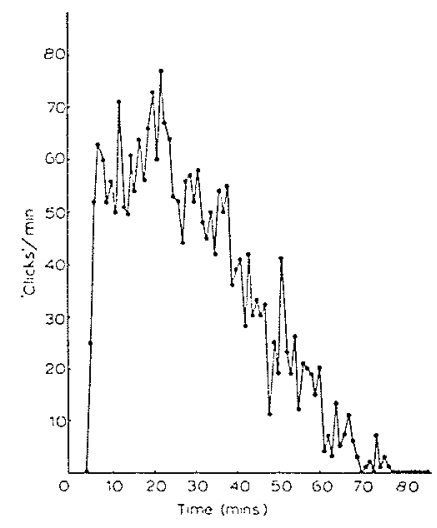

Figure 5 - Une courbe typique montrant la fréquence des "clicks" produite au cours du temps lorsqu'une feuille de Ricinus est soumise au dessèchement (d'après Milburn J.A., 1966).

Enfin, et c'est là l'expérience la plus intéressante, Milburn prend trois feuilles identiques de Ricin: la première est fraîche et récoltée le matin ; la seconde, récoltée comme la précédente subit un dessèchement puis une longue $(15 \mathrm{~h})$ réhumectation ; la troisième récoltée elle aussi le matin, subit un dessèchement puis une réhumectation rapide ( $15^{\prime}$ environ) après avoir été soumise au vide comme dans l'expérience précédente. A la suite de quoi chacune de ces trois feuilles est desséchée à nouveau et on enregistre le nombre total de "clicks" produits pendant ce dessèchement. $\mathrm{La}$ première en donne 850 , la seconde 60 , la troisième 250 . Ces faits s'interprètent bien selon Milburn, si l'on considère que seuls les vaisseaux pleins d'eau peuvent caviter. En effet dans le premier cas (feuille récoltée le matin et qu'on dessèche tout de suite) on a affaire à une feuille en parfait état c'est-à-dire dont tous les vaisseaux sont pleins d'eau. Ils cavitent donc très bien et donnent le nombre maximum de "clicks". La seconde feuille a déjà cavité pendant le premier dessèchement. La réhumectation n'a pas, malgré tout, rétabli la conduction dans tous les vaisseaux cavités, si bien que nombre d'entre eux le demeurent encore. Ils ne peuvent donc pas caviter à nouveau. D'où le petit nombre de clicks obtenus quand, une fois la réhumectation terminée, on la redessèche. Enfin la troisième a reçu le même traitement que la précédente a ceci près qu'en début de réhumectation elle a été soumise au vide (par l'intermédiaire de la pompe à vide) ce qui a bien rétabli la conduction en chassant l'air de ceux-ci. C'est pourquoi lorsqu'on la dessèche à nouveau on obtient un nombre élevé de "clicks" qui reste toutefois inférieur à celui de la première feuille n'ayant subi qu'un dessèchement.

Toutes ces expériences de Milburn montrent bien que les vaisseaux cavités offrent une grande résistance au passage de l'eau. La restauration de leur conduction s'effectue vraisemblablement en début de réhumectation, mais on ne sait quasiment rien sur sa durée. Il se peut donc que l'absence de la phase 1 de nos réhumectations corresponde aux feuilles dont les vaisseaux ont été les plus affectés par la cavitation durant le dessèchement, d'où des valeurs d'absorption à la fois bien plus faibles et très irrégulières. Par ailleurs, un autre argument en faveur du rôle de la cavitation dans les cinétiques peut être avancé. La cavitation, on le sait, augmente avec l'intensité des tensions subies par l'eau des vaisseaux, donc avec l'intensité du dessèchement. Or précisément, et contrairement à ce qu'on attendait, l'absorption initiale décroit avec l'intensité du déficit (cf. Tabl. III) ; ce qui signifie bien que la conduction dans les

\begin{tabular}{|l|c|c|c|c|}
\hline \multicolumn{4}{|c|}{ Tabsoau III } \\
en début de réhumectation (*) \\
& $\begin{array}{c}\text { Déficit } \\
10-11 \%\end{array}$ & $\begin{array}{c}\text { Déficit } \\
14-15 \%\end{array}$ & $\begin{array}{c}\text { Déficit } \\
20-21 \%\end{array}$ & $\begin{array}{c}\text { Déficit } \\
30 \%\end{array}$ \\
\hline & 339,8 & 312,5 & 276,6 & 226,6 \\
$\begin{array}{l}\text { Absorption initiale } \\
\text { moyenne (en } \\
\text { Ecart type }\end{array}$ & 85,6 & 64,7 & 88,5 & 94,5 \\
Nombre de mesures & 27 & 8 & 6 & 3 \\
\hline
\end{tabular}

(*) Correspondant à des feuilles d'une surface moyenne de $246,5 \mathrm{~cm}^{2}$ (écart type : 39,2 : nombre de mesures : 44).

vaisseaux diminue lorsque ce déficit augmente. La seconde hypothèse pourrait rendre compte d'un autre phénomène inattendu, qui se retrouve dans la majorité des cas, constituant l'autre obstacle à une modélisation simple : il se produit au cours de la réhumectation une brusque cassure, une pente plus grande succédant à une pente plus petite. Trois parametres déterminent en première approximation chacune de ces pentes : la résistance, le rapport teneur en eau/potentiel, et le volume total du compartiment correspondant. Seuls les deux premiers sont considérés comme pouvant varier dans les conditions expérimentales. Une augmentation rapide de la perméabilité à l'eau ne pouvant se traduire uniquement par une augmentation de la pente, reste le second facteur (le rapport teneur en eau/potentiel) qui croit, signifiant qu'à ce point une même quantité d'eau entraînera une chute de potentiel plus grande que précédemment, d'où la décroissance plus rapide de l'absorption. Ce fait peut s'interpréter comme une preuve soit de la non-linéarité de la relation teneur en eau/potentiel (généralement observée au niveau d'une feuille entière pour des déficits beaucoup plus importants), soit de la variation du module d'élasticité des parois en fonction de l'état de turgescence.

En résumé, l'étude de ces réhumectations a révélé que du point de vue hydrodynamique, et en dehors des conditions de flux conservatif, ou seuls certains aspects de sa structure apparaissent, la feuille de tournesol forme un système complexe.

L'utilisation des courbes pression-volume obtenues par la chambre de pression.

En 1964, Scholander redécouvrait une technique d'abord utilisée par Dixon un demi-siècle plus tôt et qui est devenue depuis l'instrument le plus utilisé pour les mesures de potentiel hydrique chez les végétaux ; le 
principe de cette technique est sommairement le suivant : lorsqu'un rameau feuillé ou même une feuille isolée est excisée d'une plante en place et transpirant, l'eau des vaisseaux ${ }^{(7)}$ qui se trouvait sous tension reflue depuis la surface coupée vers les cellules, du fait de l'arrêt de la transpiration et de l'élasticité des tissus. Ce mouvement va se poursuivre jusqu'à ce que l'interface du ménisque air-eau se trouve arrêté par les pores des parois transversales ou des ponctuations aérolées des vaisseaux. La pression hydrostatique est alors $P^{*}=2 T / r$, $T$ étant la tension de surface de l'eau des vaisseaux, $r$ le rayon des pores correspondants. S'il n'y a ni évaporation ni condensation, toute l'eau du rameau ou de la feuille est alors censée se mettre à ce même potentiel hydrique. La procédure expérimentale (Fig. 6) est la suivante : sitôt prélevé, le rameau est introduit dans la chambre de pression ou se produit pour l'eau des vaisseaux ce qui vient d'être dit. S'il n'y a pas d'espace intercellulaire isolé dans ce rameau (ce dont on ne peut être assuré), lorsque l'on monte la pression dans la chambre, l'eau va sortir des cellules et ne pourra s'échapper que par l'extrémité coupée du rameau qui pointe, par l'intermédiaire d'un joint étanche à l'air, à l'extérieur de la chambre. Au fur et à mesure que la pression dans celle-ci augmente, l'eau monte dans les vaisseaux et finit par atteindre la surface ; le rayon de courbure du ménisque devient alors très grand, $P^{\prime}$ tombe pratiquement à zéro ; le potentiel hydrique de l'eau du rameau est alors partout le même et égal à $P^{\prime}$. On aura donc, pour toute cellule :

$$
\Psi=P_{\text {total }}-\pi=P^{\prime}=0
$$

avec $\Psi=$ potentiel hydrique total, $\pi=$ potentiel osmotique
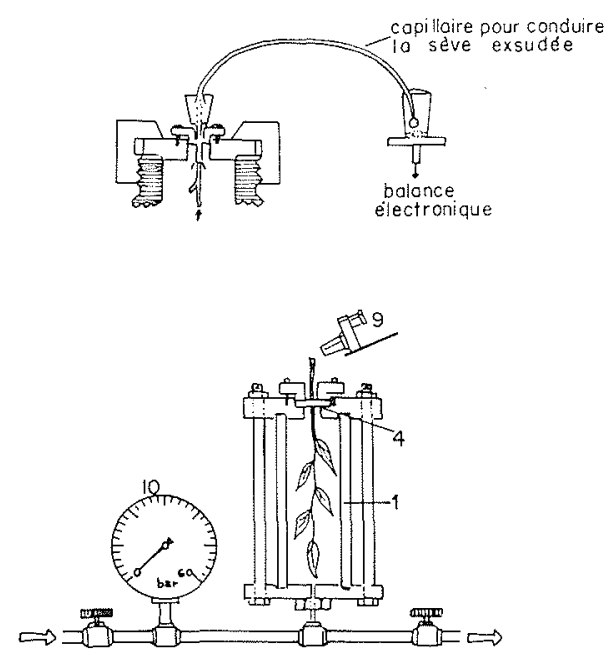

Figure 6 - Dispositif de la chambre de pression pour l'obtention des courbes pression-volume. Les organes essentiels sont : 10) le manomètre ; 1) le corps de la chambre de pression avec son dispositif de serrage 4 ; 9) la loupe binoculaire permettant de repérer l'arrivée de la sève en surface (d'après Tyree et coll., 1973).

(7) Laquelle est à une pression osmotique très faible tant que les cellules ne sont pas lésées. ou $P_{\text {total }}=P_{t}+P ; P_{t}$ étant le potentiel de turgescence et $P$, la pression dans la chambre. On a donc $P=-\Psi$. C'est là l'utilisation première et la plus courante de la chambre de pression. Mais il en est d'autres dont la plus intéressante s'appuie sur les courbes de pression-volume obtenues comme suit : si après avoir atteint l'équilibre ci-dessus défini, on accroît alors la pression de la chambre d'une valeur $\Delta P$, une petite quantité d'eau va s'écouler par la coupure, provenant des cellules, dont le potentiel hydrique va du même coup baisser. L'écoulement s'arrêtera quand la chute de potentiel correspondante sera égale à $-\Delta P$. On peut ainsi, par pas de pression, faire sortir de petits volumes successifs de sève et finalement tracer de la sorte une courbe "pressionvolume de sève extraite" en cumulant ces petites quantités (Figure 7). L'équation de cette courbe est

$$
1 / P=\left(V_{o}-V_{e}\right) /\left(R T n_{S}-F(V)\right)
$$

ou $P$ est la pression d'équilibre dans la chambre, $V_{o}$ est le volume initial de l'eau contenue dans les cellules, $V_{e}$ le volume total de sève exprimée, $n_{s}$ le nombre de moles de solutés, $F(V)$ étant égal à $\sum_{i}^{m} V_{i} P_{i}$, c'est-à-dire la somme, pour l'ensemble des cellules, des produits volumepression de turgescence, pour chaque cellule. L'exploitation "classique" de ces courbes constitue certainement à l'heure actuelle le meilleur outil d'investigations des paramètres des relations eau-feuille ou eau-rameau. Elle permet notamment d'obtenir : le volume maximal du compartiment symplastique, c'est-à-dire le volume total de l'eau contenue à l'intérieur des cellules à pleine turgescence, la pression osmotique correspondante, la valeur de cette pression osmotique en début de plasmolyse, c'est-à-dire lorsque la pression de turgescence devient nulle et, moyennant certaines précautions, le module d'élasticité des parois cellulaires. Mais on peut tenter d'aller plus loin et, comme Tyree et Coll. (1973/ $1974)^{(8)}$, travaillant sur un conifère, le Tsuga canadensis, chercher aussi à répondre à ces deux questions essentielles : combien de compartiments hydriques sont en jeu dans une feuille échangeant de l'eau avec l'extérieur ? Quelle est l'importance relative des résistances cellulaires et extra-cellulaires sur le trajet de l'eau depuis

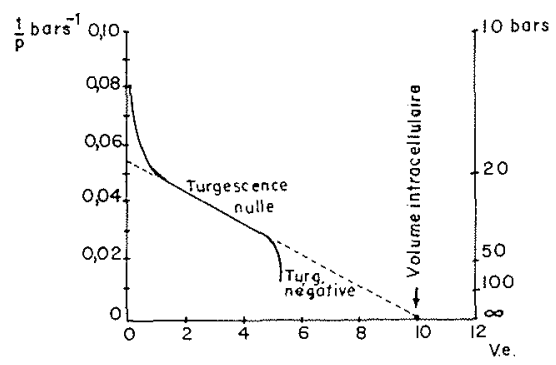

Figure 7 - Exemple de courbe pression-volume obtenue avec la chambre à pression.

(8) Leur étude est actuellement la plus approfondie du genre. 
l'extrêmité coupée d'un vaisseau jusqu'aux cellules. Les auteurs procèdent alors comme suit : ils montrent que moyennant certaines conditions (la principale étant que de très faibles variations cellulaires soient en jeu), le flux de sortie instantané d'une cellule soumise à une augmentation $\Delta P$ de pression peut s'écrire : $a(t)=\alpha \Delta P \operatorname{ex}(-\beta t) ; \alpha$ et $\beta$ étant des constantes. Ils tiennent alors le raisonnement suivant :

a) Si l'équation ci-dessus décrit le comportement d'une cellule, le flux total qui rentrera ou sortira d'un rameau formé d'une population de $m$ cellules identiques sera

$$
A(t)=\sum_{1}^{m} \alpha \Delta P \operatorname{ex}(-\beta t)
$$

b) $\mathrm{Si}$ toutes les cellules d'un rameau sont vraiment identiques, la représentation $\log A(t)=f(t)$ sera linéaire. Si toutes les cellules sont différentes, on obtiendra une courbe composée de la somme d'un grand nombre d'exponentielles décroissantes; mais si on a simplement deux ou trois groupes de cellules différentes, alors la représentation $\log A(t)$ devrait se décomposer en un nombre égal d'exponentielles décroissantes. Les auteurs en arrivent ainsi, dans le cas du Tsuga canadensis, à distinguer trois populations et moyennant d'autres hypothèses supplémentaires, ils essaient de déterminer l'importance relative des différentes résistances en jeu. Parmi les nombreuses questions que soulèvent cette étude (pour l'instant, ils n'ont pas pu répondre de façon précise aux deux questions mentionnées ci-dessus), il en est une qui me paraît très importante car elle illustre bien le rôle que la géométrie du système étudié peut jouer : quand les auteurs passent du comportement d'une cellule à celui d'une population de $m$ cellules identiques en sommant sur $m$, ils oublient de dire que cela n'est vrai que s'il existe un arrangement cellulaire en parallèle que l'on pourrait appeler ici de type additif (Pour suivre cette discussion, on se rapportera à la figure 8 ). Le système I est du type additif : les cellules (ou population de cellules identiques) sont indépendantes les unes des autres et débouchent chacune dans le même vaisseau du bois ${ }^{(9)}$. On pourra vérifier qu'à partir d'un état d'équilibre et en réponse à une augmentation $\Delta P$ de pression, l'équation d'écoulement d'une cellule dans le vaisseau répond à l'équation $a(t)=\alpha \Delta P$ ex $(-\beta t)$. Le flux total de sortie pour trois cellules identiques sera donc

$$
A(t)=\sum_{1}^{3} a(t)=3 \alpha \Delta P \operatorname{ex}(-\beta t) .
$$

d'où, en représentation semi-logarithmique, les graphes correspondants cf. Fig. 8. Le systeme II n'est plus du type additif pour le flux déversé dans le vaisseau ; il n'y a qu'une cellule qui débouche, les cellules 3 et 2 étant en série avec la première. Dans ce système, la relation ci-dessus est fausse à chaque instant. La cinétique de mise en équilibre est beaucoup plus complexe, puisque l'équation différentielle d'écoulement pour chaque cellule est du $3^{\mathrm{e}}$ ordre (cf Eq. syst. II, Fig. 8). La différence fondamentale avec le système I est que l'écoulement de chaque cellule dépend cette fois des caracté-
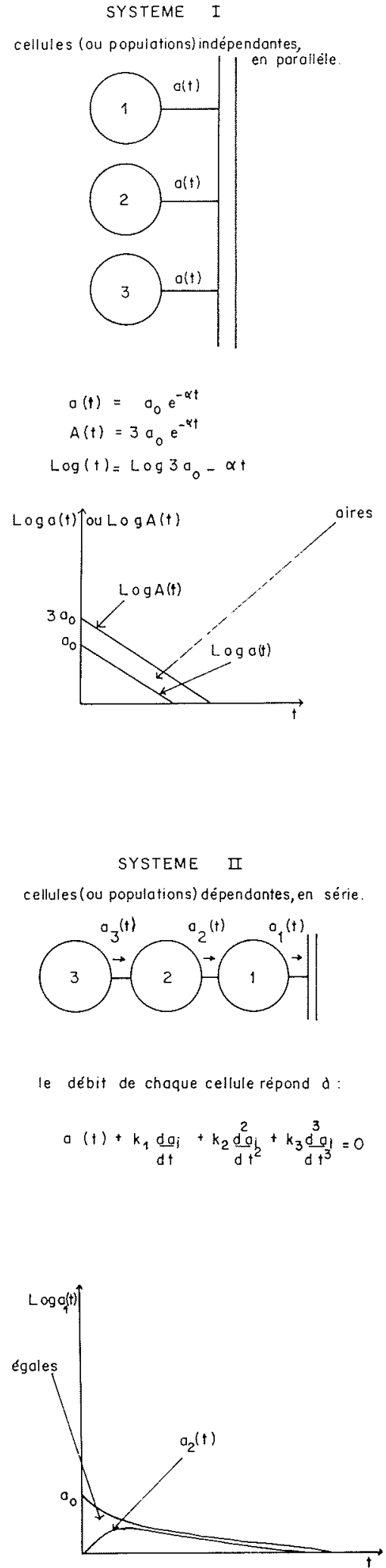

Figure 8 - Exemple de l'importance de l'arrangement des cellules lorsque l'on essaie d'appréhender le fonctionnement d'une population de cellules identiques à partir de ce qui se passe au niveau de l'une d'elles. Pour plus de détails, voir le texte.

(9) On considère que la chute de potentiel $\psi$ le long de cette portion de vaisseau est négligeable. 
ristiques de toutes les autres cellules du système et non plus des siennes seules. Par contre, si les cellules sont identiques dans les deux systèmes et l'état initial le même, l'état final sera identique dans les deux cas, c'est-à-dire que les trois cellules auront perdu chacune la même quantité d'eau à la fin. On a donc, pour le systè. me II

$$
\int_{t=0}^{t_{e q}} A(t) d t=\int_{t=0}^{t_{e q}}\left(\sum_{1}^{3} a(t)\right) d t
$$

Ainsi donc, leur proposition "Si toutes les cellules d'un même rameau sont identiques, la représentation Log $A(t)=f(t)$ sera linéaire" n'est pas du tout obligatoire. Le simple arrangement peut faire qu'il n'en soit pas ainsi. Inversement donc, le fait qu'il y ait non linéarité ne veut pas non plus dire obligatoirement que l'on ait affaire à des groupes de cellules différents. De la même façon aussi, le fait que l'on puisse bien décomposer (mais avec quelle précision sur les exponentielles $2^{\mathrm{e}}$ et $3^{\mathrm{e}}$ !), la courbe expérimentale $A(t)=f(t)$ en trois exponentielles n'est pas du tout en soi une preuve que le système que l'on décrit est du type I plutôt que II. C'est une question d'ajustement et l'on sait qu'il y en a de multiples possibles pour une courbe expérimentale.

Il faut donc rester très prudent dans l'interprétation des phases de ces cinétiques. Leur assimilation à des réservoirs différents n'est qu'une des hypothèses possibles. En particulier, on peut facilement montrer des exemples où le nombre de pentes ne correspond pas au nombre de réservoirs, deux ou plusieurs compartiments se confondent en une seule pente. Tout dépend des critères de distinction de deux compartiments et de l'échelle à laquelle on travaille. La feuille est formée d'une suite pratiquement continue de multiples petits réservoirs - les cellules -- dont l'assemblage correspond à un réseau complexe d'éléments plus ou moins différents en série et en parallèle. Au cours de la réhumectation, ce sont des zones de plus en plus étendues et éloignées le long des voies principales d'approvisionnement qui se réhumectent. Ce n'est que dans la mesure où l'ensemble des cellules d'une zone se comportent globalement différemment d'une autre zone, qu'elles peuvent être individualisées en tant que réservoirs.

\section{Comparaison}

Les deux techniques ci-dessus présentent à la fois des ressemblances (dans chaque cas il s'agit d'une cinétique de sortie ou d'entrée d'eau par le pétiole, induite par une différence de potentiel naturelle - cas de la $1^{\text {ère }}$ méthode - ou provoquée - cas de la $2^{\mathrm{e}}$ méthode -) mais aussi des différences (sens normal de la circulation de l'eau, en tension, $1^{\text {ère }}$ méth. ; sens inverse, en pression, $2^{\mathrm{e}}$ méth. ; pas de mesure de $\Psi, 1^{\text {ère }}$ méth.; une mesure par pas, $2^{\mathrm{e}}$ méth.). Jusqu'à présent, aucune comparaison n'a été entreprise sur le même matériel végétal. On ne peut donc savoir si les différences observées entre les courbes obtenues par l'une ou l'autre méthode traduisent des différences dans la nature des phénomènes observés ou bien simplement des différences quantitatives relatives aux propriétés du matériel végétal utilisé.

\section{Conclusion}

Les hydrodynamiciens présents ont sans doute été surpris, sinon déçus, du peu de références fait à leur discipline - la mécanique des fluides - dans l'étude des relations plante-eau et encore plus peut-être, du niveau élémentaire de ces références. Il ne faudrait pas croire que tous les travaux s'en tiennent à ce stade. En particulier, les études des propriétés hydriques des bois font appel à un arsenal plus poussé mais qui fait aussi, il est vrai, une large place à la thermodynamique. Cependant à côté de raisons historiques, il en est d'autres, non pour justifier mais au moins pour expliquer en partie cet état de fait : la méconnaissance des différentes caractéristiques hydrodynamiques de l'architecture vasculaire, la finesse des éléments conducteurs, l'état de tension et non de pression de l'eau dans ces éléments, la complexité du réseau, le problème de la présence de deux phases, liquide et vapeur, de membranes biologiques presque partout associées (liaison xylème-phloeme) et par ailleurs la pauvreté et l'inadequation des techniques de mesures. On peut espérer que cette rencontre change quelque peu cette situation grâce à la présence tant des biophysiciens travaillant sur la circulation sanguine que des hydrodynamiciens. En attendant leurs propres réflexions, critiques et suggestions, voici de notre côté, parmi d'autres, quelques propositions :

a) La cavitation : concernant la cavitation, le problème est le suivant : Quelle est son importance chez les végétaux supérieurs dans les conditions naturelles (en particulier dans les périodes de forte évapotranspiration et de dessèchement). Il ne s'agit pas pour nous de s'engager dans une description physique du phénomène - lequel doit être fort complexe a l'intérieur des structures telles que les vaisseaux du bois - mais de pouvoir en apprécier l'importance par une mesure quantitative ou semi-quantitative. Dans ce sens, Milburn a développé une méthode fondée sur l'enregistrement après amplification des ultrasons produits lors des phénomènes de cavitation.

b) Comme il me semble que le but poursuivi par ceux qui s'occupent des relations plante eau au niveau de l'individu plante est en particulier de comprendre quelles sont les règles de fonctionnement de cet organisme hiérarchisé formé de surfaces absorbantes et transpirantes inter-connectées entre elles, et non pas seulement de donner des valeurs (variables) de résistances, il se pose la question de savoir comment aborder l'étude de la circulation dans un réseau complexe aux bornes duquel existent des conditions variables. Il me semble que, d'une part les médecins a propos de la circulation dans des réseaux de capillaires aux propriétés diverses, d'autre part les hydrologues dans l'étude de la circulation en milieu poreux ou sur des réseaux hydrologiques complexes, ont développé des concepts, des modeles ou des méthodes de simulation qui pourraient nous être fort utiles.

c) Enfin, du point de vue des techniques de mesure - de flux liquides en particulier - il est certain qu'un échange approfondi, ou même une collaboration, pourraient être utiles, soit pour faire connaître l'instrumentation employée (les médecins ont certainement des 
procédés intéressants), soit pour en trouver une meilleure (la magnétohydrodynamique a déjà fourni, semblet-il, un moyen de connaître avec une bonne précision le flux circulant dans des tiges de faibles diamètres).

\section{Bibliographie}

CRUIZIAT P. (1972). - Contribution à l'étude des réserves en eau de la plante. Thèse de docteur de $3^{\mathrm{e}}$ cycle. Paris VI, 82 p. ; 43 Fig.

CRUIZIAT P., BODET C. (1974). - Détermination des pertes en eau subies par les différents organes d'une plante soumise au dessèchement. Ann. Agro. 25 (4) p. 539-554.

DIXON H., H. (1914). - Transpiration and the ascent of the sap in plants. The Macmillan Company, New York.

HUCK M.G., KLEPPER B., TAYLOR M., M. (1970). - Diurnal variations in root diameter. Plant. Physiol., 45, 529-530.

JARVIS P., G. (1975). - Water transfer in plants in "Heat and Mass transfer in the biosphere". I. Transfer processes in plant environment. Edit by D.A. de Vries and N.H. Afgan. John Wiley \& sons, New-York, p. 369-394.

KRAMER P.J. (1938). - Root resistance as the cause of the absorption lag. Amer. J. Bot. 25, p. 110-113.

MILBURN J.A. (1966 a). - The conduction of sap. I. Water conduction and cavitation in water stressed leaves. Planta (Berl.) $69: 34-42$.
MILBURN J.A. (1966 b). - The conduction of sap. II. Detection of vibrations produced by sap cavitation in Ricinus Xylem. Planta (Berl.) $69:$ 43-52.

MILBURN J.A. (1973 a). - Cavitation in Ricinus by acoustic detection ; induction in excised leaves by various factors. Planta (Berl.) $110: 253-264$.

MILBURN J.A. (1973 b). - Cavitation studies on whole Ricinus plants by acoustic detection. Planta (Berl.) 112:333-342.

MILBURN J.A., McLAUGHLIN M.E. (1974). - Studies of cavitation in isolated vascular bundles and whole leaves of Plantago major. New Phytol. $73: 861-871$.

SCHOLANDER P.F., HAMMEL H.T., HEMMINGSEN E.A. and BRADSTREET E.D. (1964). - Hydrostatic pressure and osmotic potential in leaves of mangroves and some other plants. Proc. nat. Acad. Sci. U.S.A., 52, 119-125.

TYREE M.T., DAINTY J. and DENIS M. (1973). - The water relations of hemlock, (Tsuga canadensis). I. Some equilibrium water relations as mesured by the pressure-bomb technique. Can. J. Bot. $51: 1471-1480$.

TYREE M.T. and DAINTY J. (1973), - The water relations of hemlock (Tsuga canadensis) II. The kinetics of water exchange between the symplast and apoplast. Can. J. Bot. $51: 1481-1489$.

TYREE M.T., CADWELL C. and DAINTY J. (1974). - The water relations of hemlock (Tsuga canadensis) V. The localization of resistances to bulk water flow. Can. J. Bot. 53 : $1078-1084$.

WEST D.W. and GAFF D.F. (1976). - Xylem cavitation in excised leaves of Malus Sylvestris Mill. and measurement of leaf water status with pressure chamber. Planta (Berl.) $129: 15-18$

Discussion

Président : M.S. HENIN

Le Président remercie M. CRUIZIAT de son exposé et il ouvre la discussion :

M. PERRIER évoque le problème des régimes transitoires : M. CRUIZIAT définit un régime permanent par la formule: absorption $=$ transpiration. Ce n'est vrai que sur un très grand intervalle de temps.

Mais entre cette entrée et cette sortie, il y a tout un monde extrêmement complexe. Tout en ayant absorption = transpiration, on peut donc avoir un grand nombre de variations et divers régimes transitoires à l'intérieur même des différentes parties de la plante. Je crois que c'est là une des grandes raisons de nos difficultés expérimentales; beaucoup d'expériences d'absorption transpiration donnent des résultats extrêmement divers parce qu'on oublie qu'au sein de la plante on a des régimes transitoires.

M. CRUIZIAT a dit que, sur un court intervalle de temps - de l'ordre de 10 minutes - on a un régime permanent. Je pense qu'on n'a jamais un régime permanent avec une plante ; on l'a peut-être en fin de nuit, si toutes les conditions sont favorables et encore faudrait-il le vérifier.

M. COMOLET remarque que la cavitation ne semble prati- quement jouer aucun rôle. Il évoque ensuite le flétrissement où il voit une perte d'eau se traduisant par une contraction générale.

M. CRUIZIAT confirme, à sa demande, que la géométrie diminue globalement tout en précisant qu'il n'est pas certain que le bois et les cellules extérieures se contractent de la même façon. Le flétrissement, ajoute M. CRUIZIAT, est essentiellement une perte de la pression de turgescence. C'est comme une chambre à air : quand la pression diminue, cela s'affaisse.

M. COUCHAT rappelle certaines applications des traceurs dans les milieux inertes. Peut-on envisager, poursuit-il, dans le simple cadre d'une feuille par exemple, d'introduire un traceur à l'entrée de l'eau, au niveau du pétiole, et d'analyser la courbe à la sortie de ce traceur, tritium par exemple? Ne peut-on pas essayer d'en déduire quelque chose sur la structure?

M. CRUIZIAT croit savoir que des expériences un peu similaires ont été conduites. Mais il est vraiment très difficile, pense-t-il, d'obtenir des informations aussi fines qu'on le souhaiterait dans un milieu aussi hétérogène que la feuille avec - complication supplémentaire - passage de la phase liquide à la phase vapeur. 